\title{
On boundary behaviour of the Bergman projection on pseudoconvex domains
}

\author{
by \\ M. JASICZAK (Poznań)
}

\begin{abstract}
It is shown that on strongly pseudoconvex domains the Bergman projection maps a space $L v_{k}$ of functions growing near the boundary like some power of the Bergman distance from a fixed point into a space of functions which can be estimated by the consecutive power of the Bergman distance. This property has a local character.

Let $\Omega$ be a bounded, pseudoconvex set with $C^{3}$ boundary. We show that if the Bergman projection is continuous on a space $E \supset L^{\infty}(\Omega)$ defined by weighted-sup seminorms and equipped with the topology given by these seminorms, then $E$ must contain the spaces $L v_{k}$ for each natural $k$. As a result, in the case of strongly pseudoconvex domains the inductive limit of this sequence of spaces is the smallest extension of $L^{\infty}$ in the class of spaces defined by weighted-sup seminorms on which the Bergman projection is continuous. This is a generalization of the results of J. Taskinen in the case of the unit disc as well as of the previous research of the author concerning the unit ball.
\end{abstract}

1. Introduction. Let $\Omega$ be a bounded domain in $\mathbb{C}^{n}$. The Bergman projection $B: L^{2}(\Omega) \rightarrow L^{2}(\Omega)$ is the orthogonal projection onto the closed subspace $H^{2}(\Omega)$ of square integrable holomorphic functions on $\Omega$. The projection $B$ can be represented as an integral operator

$$
B f(z)=\int_{\Omega} f(\zeta) K_{\Omega}(z, \zeta) d V(\zeta) .
$$

The function $K_{\Omega}(z, \zeta)$, the Bergman kernel, belongs to $L^{2}$ for each fixed $z \in \Omega$ so that the integral is well defined.

It is an immediate consequence of the definition that the Bergman projection is bounded on $L^{2}$. However, the situation on other $L^{p}$, Lipschitz or Sobolev spaces is more subtle. As might be expected, this subject has been widely investigated. An archetypical result here is the Forelli-Rudin theorem in the unit ball $\mathbb{B}$ in $\mathbb{C}^{n}$, according to which $B$ is bounded on $L^{p}(\mathbb{B})$ provided $1<p<\infty$. In fact, [16] also investigated the problem of continuity of other Bergman type projections, but this subject will not be dealt with here.

2000 Mathematics Subject Classification: 32A25, 32A36, 32A40, 32A70, 32T15, 32T40, $32 \mathrm{~F} 45$. 
It is known that in the case of strongly pseudoconvex domains the Bergman projection is bounded on Lipschitz spaces [2]. Significantly, continuity on Sobolev spaces on strongly pseudoconvex domains (generally, domains satisfying condition (R)) proved crucial to understanding the boundary behaviour of biholomorphic mappings [9]. On the other hand, it is known that some other classes of functions such as $C^{k}$ and $\operatorname{Lip}_{k}$ are not preserved by $B$.

Investigation of $L^{p}$ and Hölder boundedness of $B$ as well as continuity on Sobolev spaces requires an exact knowledge of the singularities of the Bergman kernel. The case of strongly pseudoconvex domains is now well understood due to profound theorems of Fefferman, Boutet de Monvel and Sjöstrand describing the asymptotic behaviour of the Bergman kernel. It is worth mentioning that the situation of weakly pseudoconvex domains is still elusive. The aforementioned theorem of Fefferman, Boutet de Monvel and Sjöstrand is also crucial to our study.

We concentrate on the behaviour of the Bergman projection on the space of bounded measurable functions, or equivalently, by duality, on $L^{1}$. From the Forelli-Rudin theorem it follows that $B$ does not map $L^{\infty}(\mathbb{B})$ into itself. It is a conclusion of our study that to maintain continuity of $B$ one has to change a way of thinking what boundedness of a function means.

We will show that on strongly pseudoconvex bounded domains with smooth boundary the Bergman projection preserves the space of functions growing near the boundary like some power of the Bergman distance $b\left(z_{0}, \cdot\right)$ from a fixed point (equivalently, like the Carathéodory, or the Kobayashi distance or, as will be shown, like the logarithm of the distance to the boundary). In fact, we will prove a result which in brief can be summed up as a shift in log growth of the image of the Bergman projection on strongly pseudoconvex domains.

In view of [11] and Grothendieck's factorization theorem (see [29]), this is tantamount to proving continuity of $B$ on this space equipped with the inductive topology of a sequence of Banach spaces $L v_{n}$. The space $L v_{n}$ consists of measurable functions which can be estimated by the Bergman distance from a fixed point.

Equivalently, the result can be summarized as finding the smallest weighted-sup extension of the space of bounded measurable functions equipped with the topology given by the weighted-sup seminorms, on which the Bergman projection is continuous. This was the starting point for [30] in the unit disc $\mathbb{D}$ as well as in [22] in the case of the unit ball, where we have formulated the problem of extending the result to strongly pseudoconvex domains.

Interestingly, the problem of global and local regularity does not have to be equivalent for $B$. This is a consequence of a striking result in [3], where it 
was shown that failure of regularity of $B$ at any finite level of differentiability must stem from global considerations.

We will also prove a local version of the aforementioned results. We show that if $\omega \in \partial \Omega$ is strictly Levi pseudoconvex, then there exists an open set $\mathcal{U}_{\omega} \ni \omega$ such that for each measurable function $f \in L^{2}(\Omega)$ satisfying $|f(z)| \leq C b\left(z_{0}, z\right)^{m}$ for some $m \in \mathbb{N}$ on $\mathcal{U}_{\omega} \cap \Omega$, we have

$$
|B f(z)| \leq C^{\prime} b\left(z_{0}, z\right)^{m+1}, \quad z \in \widetilde{\mathcal{U}}_{\omega} \cap \Omega,
$$

where $C, C^{\prime}$ are positive constants, $\widetilde{\mathcal{U}}_{\omega} \subset \subset \mathcal{U}_{\omega}$ and $z_{0}$ is a fixed point in $\Omega$. We assume that the domain is pseudoconvex of finite type with a smooth boundary. This result can again be translated into continuity on some subspaces of $L^{2}$.

Making use of Henkin's construction of peak functions and Bell's operator $\Phi$, we will show that if $\omega$ is a strongly pseudoconvex point of $\partial \Omega$ then there always exists a function $f_{m}$ belonging to $H^{2}$ which grows exactly like $\left|\log \operatorname{dist}_{\Omega}(z)\right|^{m}$ as $z \rightarrow \omega$, where $m$ is a natural number. The function $f_{m}$ is the image under $B$ of a function (namely $\Phi f_{m}$ ) which can be estimated by $\left|\log \operatorname{dist}_{\Omega}(z)\right|^{m-1}$. As a result, the description of the asymptotic behaviour of $B$ near a strongly pseudoconvex piece of the boundary in the above theorem is the best possible. Correspondingly, we also prove that the inductive limit of a sequence of spaces consisting of functions growing like consecutive powers of the Bergman distance is the smallest weighted-sup extension of $L^{\infty}$ equipped with the topology given by the weighted-sup seminorms, on which $B$ is continuous.

Recall that a smooth bounded domain $\Omega$ satisfies condition (R) if $B$ preserves $C^{\infty}(\bar{\Omega})$ or equivalently for each positive integer $s$ there is an integer $M_{s}$ such that $B$ is bounded from $W_{0}^{s+M_{s}}$ to $H^{s}$ (for definitions and proofs see [8]). By [7] condition ( $\mathrm{R}$ ) is tantamount to preserving the space of functions growing like some power of $\operatorname{dist}_{\Omega}(z)$. A more than formal similarity of our results to this condition is worth noticing. Thus, it seems to be of interest to understand the relation between the two observations.

As stated before, the following theorem ([15], [12]) is crucial to our study.

Theorem 1 (Fefferman, Boutet de Monvel, Sjöstrand). Let $\Omega$ be $a$ bounded strongly pseudoconvex domain in $\mathbb{C}^{n}$ with smooth, non-degenerate real-valued defining function $\varrho$. There exist functions $F, G \in C^{\infty}(\bar{\Omega} \times \bar{\Omega})$ and $\psi \in C^{\infty}\left(\mathbb{C}^{n} \times \mathbb{C}^{n}\right)$ such that

$$
K_{\Omega}=F(-i \psi)^{-n-1}+G \log (-i \psi) .
$$

The function $\psi$ satisfies the following conditions:

(i) $\psi(z, z)=i^{-1} \varrho(z)$,

(ii) $\psi(z, \zeta)=-\overline{\psi(\zeta, z)}$, 
(iii) $\operatorname{Im} \psi(z, \zeta) \geq C\left(\delta_{\Omega}(z)+\delta_{\Omega}(\zeta)+|z-\zeta|^{2}\right)$ for some positive constant $C$.

(iv) $\psi$ is almost analytic in $z, \bar{w}$ in the sense that $\bar{\partial}_{z} \psi(z, w)$ and $\partial_{w} \psi(z, w)$ vanish to infinite order at $z=w$.

We denote most constants by $C$; their values may change from line to line.

REMARK. Some results from this paper (Corollaries 6 and 12) were announced during the Conference on Smooth and Analytic Spaces, Będlewo, April 2003. At that time the author was informed by J. Taskinen that he and M. Engliš were working on similar problems. During a visit in Prague (May 2003) the author received a preprint of their results [14].

2. Preliminaries. Let $\Omega$ be a bounded domain in $\mathbb{C}^{n}$ given by a smooth defining function $\varrho$. We tacitly assume that $d \varrho \neq 0$ on $\partial \Omega$. Consequently, the boundary of $\Omega$ is a smooth manifold in $\mathbb{C}^{n}$. Although the next lemma is obvious we include it for completeness.

Lemma 2. Assume that $\partial \Omega=\{\varrho=0\}$, where $\varrho$ is $C^{2}$ and $d \varrho \neq 0$ on $\partial \Omega$. There is an open cover $U_{1}, \ldots, U_{l}$ of $\partial \Omega$ and positive numbers $\epsilon, \gamma$ such that for each $k, 1 \leq k \leq l$, there exists an index $j, 1 \leq j \leq n$, such that

$$
\left|\frac{\partial \varrho}{\partial \zeta_{j}}\right| \geq \gamma
$$

on the $\epsilon$-neighbourhood $\left(U_{k}\right)_{\epsilon}$ of $U_{k}$.

Recall also that, since the boundary is $C^{k}, k \geq 2$, the function defined by

$$
\varrho(z)= \begin{cases}-\operatorname{dist}(z, \partial \Omega), & z \in \Omega, \\ \operatorname{dist}(z, \partial \Omega), & z \notin \Omega,\end{cases}
$$

is a $C^{k}$ defining function of $\Omega$. If no confusion occurs, we write simply $\delta_{\Omega}$ to denote the distance $\operatorname{dist}(z, \partial \Omega)$ of $z$ to the boundary of $\Omega$.

From the fact that $\partial \Omega$ is $C^{k}, k \geq 2$, it follows that there exists an open set $U \supset \partial \Omega$ and a function $\pi: U \rightarrow \partial \Omega$ such that $\pi(\omega)=\omega$ for $\omega \in \partial \Omega$ and $\pi^{-1}(\omega)$ is a curve in $U$ which intersects the boundary transversally at $\omega$. The function $\pi$ is called a projection. Recall that

$$
c|\varrho(z)| \leq|\pi(z)-z| \leq C|\varrho(z)|
$$

for some positive constants $c, C$ and $z \in U$.

Now we turn to the functional-analytic background.

Definition 1. A continuous function $v:(0,1) \rightarrow \mathbb{R}_{+}$is said to be a weight if for each $k \in \mathbb{N}$,

$$
\sup _{r \in(0,1)} v(r)|\log r|^{k}<\infty .
$$


The set of all weights will be denoted by $W$. We define $v_{k}: \mathbb{R}_{+} \rightarrow \mathbb{R}$ by

$$
v_{k}(r)= \begin{cases}|\log r|^{-k} & \text { if } r<e^{-1} \\ 1 & \text { if } r \geq e^{-1}\end{cases}
$$

It should be emphasized that by a weight we sometimes mean the composition $v \circ|\varrho(\cdot)|$, where $\varrho$ is a defining function.

Without loss of generality we may assume that $\sup _{z \in \Omega}|\varrho(z)|<1$. Let $v:(0,1) \rightarrow \mathbb{R}_{+}$be a continuous function. By a weighted-sup seminorm we mean a seminorm of the form

$$
\|f\|_{v}=\sup _{z \in \Omega}|f(z)| v(|\varrho(z)|) .
$$

Thus we restrict our attention to the radial case.

The symbol $\mathcal{U}$ will stand for any fixed, open set such that $\mathcal{U} \cap \partial \Omega \neq \emptyset$. We will assume in the next section that $\mathcal{U} \cap \partial \Omega$ is contained in a strongly Levi pseudoconvex piece of the boundary of $\Omega$.

Definition 2. $L W_{\mathfrak{U}}(\Omega)$ is the space of measurable functions $f: \Omega \rightarrow \mathbb{C}$ such that for each $v \in W$,

$$
\|f\|_{\mathcal{u}, v}=\|f\|_{L^{2}(\Omega)}+\sup _{z \in \mathcal{U} \cap \Omega}|f(z)| v(|\varrho(z)|)<\infty .
$$

We simply write $L W$ if $\mathcal{U} \supset \bar{\Omega}$. Let $L v_{k, \mathcal{U}}(\Omega)$ denote the space of functions satisfying

$$
\|f\|_{k, \mathcal{U}}=\|f\|_{L^{2}(\Omega)}+\sup _{z \in \mathcal{U} \Omega}|f(z)| v_{k}(|\varrho(z)|)<\infty .
$$

If $\mathcal{\supset} \bar{\Omega}$ and no confusion occurs, we simply write $L v_{k}$.

The symbols $H W_{\mathcal{U}}(\Omega), H v_{k, \mathcal{U}}(\Omega), H W, H v_{k}$ will stand for the corresponding spaces of functions holomorphic in $\Omega$.

Basically, the space $L W_{\mathfrak{U}}(\Omega)$ consists of functions which are square integrable on the whole $\Omega$ and bounded a.e. on $\mathcal{U}$ after multiplying by each weight. Observe that $L v_{k, u}$ is a Banach space. Indeed, let $\left(f_{n}\right)$ be a Cauchy sequence in $L v_{k}, \mathcal{u}$. Since $\left(f_{n}\right)$ is a Cauchy sequence in $L^{2}(\Omega)$ there exists $f \in L^{2}(\Omega)$ such that $f_{n}$ tends to $f$ in $L^{2}(\Omega)$. On the other hand, $f_{n} \mid u$ is a Cauchy sequence in

$$
\left\{f: \sup _{\mathcal{U} \cap \Omega}|f| v_{k}<\infty\right\}
$$

which is a Banach space. Consequently, there exists a measurable function $g$ on $\mathcal{U}$ which is the limit of $f_{n}$ on $\mathcal{U}$. Now, it suffices to show that $\left.f\right|_{\mathcal{U}}=g$; but this follows from the estimate

$$
\left\|\left.f\right|_{\mathfrak{u}}-g\right\|_{L^{2}(\mathcal{u})} \leq\left\|f-f_{n}\right\|_{L^{2}(\Omega)}+C \sup _{\mathfrak{u}}\left|f_{n}\right| u-g \mid v_{k} .
$$

Similarly, one shows that the corresponding spaces of holomorphic functions are Banach spaces. From the projective description of weighted in- 
ductive limits ([11]) it follows that $H W(\Omega)$ is the inductive limit of the compact sequence $\left(H v_{n}\right)$. Thus $H W(\Omega)$ is a Silva space (in the literature, Silva spaces are also called (DFS)-spaces). Therefore, $H W$ is a complete reflexive Schwartz space and hence Montel. Furthermore, $H W$ carries the finest topology which makes all injections $\iota_{k}: H v_{k} \hookrightarrow H W$ continuous (not only the finest locally convex topology).

The dual projective sequence $\left(H v_{n}\right)_{\mathrm{b}}^{\prime}$ is compact and its limit is a reflexive Fréchet space (an excellent reference book is [29]; see also the survey [10] and $[22])$.

Recall that the classical Forelli-Rudin theorem ([16]) says that

$$
\left|B\left(|\varrho(\cdot)|^{-\alpha}\right)\right| \leq C|\varrho(\cdot)|^{-\alpha}
$$

for $0<\alpha<1$, where $\varrho(z)=|z|^{2}-1$ is a defining function for the unit ball. This statement is just continuity with respect to a weight of the form $v(t)=t^{\alpha}$. The next lemma, with an easy proof in [22], allows us to reduce the case of each weight to this particular one.

Lemma 3. Let $v \in W$ and $\alpha>0$.

(i) The function $w(r)=\sup _{s \leq r} v(s)$ belongs to $W$.

(ii) Define

$$
v_{\beta}(r)= \begin{cases}v(r) & \text { if } r \geq \beta, \\ v(\beta) \beta^{-\alpha} r^{\alpha} & \text { if } r<\beta .\end{cases}
$$

Then the function $w(r)=\max \left\{\sup _{0<\beta<1 / 2} v_{\beta}(r), v(r)\right\}$ is a weight.

(iii) For each $\alpha>0$, the function $v^{\alpha}$ is an element of $W$.

As stated in the introduction, we intend to localize our results. Namely, we prove that the log shift is typical for the Bergman projection near each point such that the Levi form is positive definite on the complex tangent space. The most important tool is the following proposition.

Proposition 4 ([13]). Let $\Omega_{1} \subset \Omega$ be two pseudoconvex domains with $C^{\infty}$ boundaries and $U$ a neighbourhood of a point $z_{0} \in \partial \Omega$ such that $U \cap \partial \Omega_{1}=U \cap \partial \Omega$ and the piece of the common boundary is strongly pseudoconvex. Then the difference $K_{\Omega_{1}}(z, \zeta)-K_{\Omega}(z, \zeta)$ is $C^{\infty}$ on $\left(U \cap \bar{\Omega}_{1}\right) \times \bar{\Omega}_{1}$.

Observe that from the description of the asymptotic behaviour of the Bergman kernel given by Fefferman, Boutet de Monvel and Sjöstrand it follows that the Bergman kernel of a strongly pseudoconvex domain is bounded off the set

$$
\Delta_{\varepsilon}=\left\{(z, \zeta) \in \Omega \times \Omega: \delta_{\Omega}(z) \geq \varepsilon, \delta_{\Omega}(\zeta) \geq \varepsilon,|z-\zeta| \geq \varepsilon\right\}
$$

by a constant independent of $z, \zeta$. It is worth mentioning that for $C^{\infty}$ do- 
mains in $\mathbb{C}$ this fact is a consequence of the equality

where $G$ is the Green function.

$$
K(z, \zeta)=-\frac{2}{\pi} \frac{\partial^{2} G(z, \zeta)}{\partial z \partial \bar{\zeta}}
$$

It was shown by N. Kerzman in [23] that for a bounded strongly pseudoconvex domain $\Omega$ with smooth boundary the Bergman kernel $K(\cdot, \cdot)$ is smooth in $(\bar{\Omega} \times \bar{\Omega}) \backslash \Delta$, where $\Delta$ is the diagonal in $\partial \Omega \times \partial \Omega$. The same proof works for domains of finite type [25], [26] (for definition see [25], [28]). We will repeatedly refer to this result in the proof of our theorem on the regularity of the Bergman projection.

We also use local versions of well-known results on the asymptotic behaviour of the Carathéodory, Kobayashi and Bergman distances. This might be a proper language to generalize the properties of the Bergman projection to a broader class of domains.

We refer the reader to [24] and [21] for the definitions and properties of invariant metrics and distances. The symbols $c_{\Omega}\left(z_{0}, z\right), b_{\Omega}\left(z_{0}, z\right), k_{\Omega}\left(z_{0}, z\right)$ stand for the Carathéodory, Bergman and Kobayashi distances of $z_{0}$ and $z$, respectively.

3. Results. Let $\Omega$ be a bounded domain of finite type in $\mathbb{C}^{n}$. Assume that $\Omega$ is given by a $C^{\infty}$ defining function $\varrho$ which is plurisubharmonic in a neighbourhood $\mathcal{O}$ of $\partial \Omega$. By $\mathcal{O}_{\mathrm{s}}$ we denote the set of points $z$ in which $\varrho$ is strictly plurisubharmonic, i.e.

$$
\sum_{j, k=1}^{n} \frac{\partial^{2} \varrho}{\partial z_{j} \partial \bar{z}_{k}}(z) \zeta_{j} \bar{\zeta}_{k}>0
$$

for each $\zeta \in \mathbb{C}^{n}, \zeta \neq 0$. The symbol $\mathcal{U}$ will stand for any fixed bounded open set which is a relatively compact subset of $\mathcal{O}_{\mathrm{s}}$. From the assumptions it follows that $\Omega$ is pseudoconvex and $\partial \Omega \cap \mathcal{O}_{\mathrm{s}}$ is a strongly Levi pseudoconvex piece of its boundary. This set is denoted by $(\partial \Omega)_{\mathrm{s}}$. An obvious example of a domain satisfying these conditions which is not strongly pseudoconvex is the set of points $\left(z_{1}, z_{2}\right)$ in $\mathbb{C}^{2}$ such that

$$
\left|z_{1}\right|^{2}+\left|z_{2}\right|^{4}<1
$$

ThEOREM 5. Assume that $\Omega$ satisfies the above conditions. Then the Bergman projection is a continuous operator from $L W_{\mathfrak{U}}(\Omega)$ to $H W_{\widetilde{u}}(\Omega)$, where $\tilde{U} \subset \subset \mathcal{U}$.

An immediate consequence of this theorem is the following

Corollary 6. Assume that $\Omega$ is a bounded, strongly pseudoconvex domain in $\mathbb{C}^{n}$ with a smooth boundary. Then the Bergman projection is continuous on $L W(\Omega)$. 
As mentioned before, we will reduce the general case to the case of weights of the form $t^{1 / 2}$. Thus the following generalization of the ForelliRudin theorem is of interest.

Lemma 7. For $\Omega$ satisfying the assumptions of Theorem 5 there exists $\epsilon$ such that for $z \in \Omega$ with $\delta_{\Omega}(z)<\epsilon$ we have

(i) $\int_{u \cap \Omega} \frac{|K(z, \zeta)|}{\left|\varrho^{\alpha}(\zeta)\right|} d V(\zeta) \leq C|\varrho(z)|^{-\alpha}, \quad 0<\alpha<1$,

(ii) $\int_{\text {uก } \Omega}|K(z, \zeta)| d V(\zeta) \leq C|\log | \varrho(z)||$.

Proof. Since by assumption $\mathcal{U} \subset \subset \mathcal{O}_{\mathrm{s}}$, there exists $\epsilon>0$ such that for each $\omega \in \partial \Omega \cap \mathcal{U}$ the ball $B(\omega, 2 \epsilon)$ is contained with its closure in $\mathcal{O}_{\mathrm{s}}$. If $z \in \Omega$ and $\delta_{\Omega}(z)<\epsilon$, then obviously $B\left(z, \delta_{\Omega}(z)\right) \subset B(\pi(z), 2 \epsilon)$ and $B\left(z, \delta_{\Omega}(z)\right)$ is contained in $\mathcal{O}_{\mathrm{s}}$. Denote by $B_{c}$ a ball satisfying $B(\pi(z), 2 \epsilon) \subset \subset$ $B_{c}=B(\pi(z), c) \subset \mathcal{O}_{\mathrm{s}}$. Choose an increasing $C^{\infty}$ function $\chi:[0, c) \rightarrow \mathbb{R}$ such that $\chi \equiv 0$ on $\left[0,4 \epsilon^{2}\right], \chi^{\prime \prime} \geq 0$ on $\left[4 \epsilon^{2}, c\right)$ and $\lim _{t \rightarrow c-} \chi(t)=\infty$. Define

$$
\Omega_{1}=\left\{\zeta \in \mathbb{C}^{n}: \varrho_{1}(\zeta)=\varrho(\zeta)+\chi\left(|\zeta-\pi(z)|^{2}\right)<0\right\} .
$$

Straightforward calculations show that $\Omega_{1} \subset \Omega$ is a strongly pseudoconvex domain such that $B(\pi(z), 2 \epsilon) \cap \partial \Omega_{1}=B(\pi(z), 2 \epsilon) \cap \partial \Omega$.

Take $\varepsilon>0$ such that a ball $B_{\varepsilon}=B(z, \varepsilon) \subset B(\pi(z), 2 \epsilon)$ is tangent to $B(\pi(z), 2 \epsilon)$. It is important to notice that $\varepsilon$ is bounded below by $\epsilon$ for each $z \in \mathcal{U}$. From Proposition 4 it follows that the difference $K_{\Omega}-K_{\Omega_{1}}$ is smooth in $\left(B(\pi(z), 2 \epsilon) \cap \bar{\Omega}_{1}\right) \times \bar{\Omega}_{1}$. Consequently,

$$
\begin{aligned}
\int_{u \cap \Omega} \frac{\left|K_{\Omega}(z, \zeta)\right|}{|\varrho(\zeta)|^{\alpha}} d V(\zeta)= & \left\{\int_{u \cap B_{\varepsilon} \cap \Omega}+\int_{u \cap \Omega \backslash B_{\varepsilon}}\right\} \frac{\left|K_{\Omega}(z, \zeta)\right|}{|\varrho(\zeta)|^{\alpha}} d V(\zeta) \\
\leq & C_{\varepsilon} \int_{\Omega} \frac{d V(\zeta)}{|\varrho(\zeta)|^{\alpha}}+\int_{u \cap B_{\varepsilon} \cap \Omega} \frac{\left|K_{\Omega_{1}}(z, \zeta)\right|}{|\varrho(\zeta)|^{\alpha}} d V(\zeta) \\
& +\int_{u \cap B_{\varepsilon} \cap \Omega} \frac{\left|K_{\Omega}(z, \zeta)-K_{\Omega_{1}}(z, \zeta)\right|}{|\varrho(\zeta)|^{\alpha}} d V(\zeta) \\
\leq & C_{\varepsilon}+\int_{u \cap B_{\varepsilon} \cap \Omega} \frac{\left|K_{\Omega_{1}}(z, \zeta)\right|}{\left|\varrho_{1}(\zeta)\right|^{\alpha}} d V(\zeta) .
\end{aligned}
$$

In the last line we have used the fact that $\varrho(\zeta)=\varrho_{1}(\zeta)$ for $\zeta \in \Omega \cap B(\pi(z), 2 \epsilon)$. Therefore, it is enough to estimate the integral

$$
\int_{\Omega_{1}} \frac{\left|K_{\Omega_{1}}(z, \zeta)\right|}{\left|\varrho_{1}(\zeta)\right|^{\alpha}} d V(\zeta),
$$

where now $\Omega_{1}$ is a strongly pseudoconvex domain with a defining function $\varrho_{1}$, which will again be denoted by $\varrho$. 
Part (i) of the lemma is proved in [2, Lemma 2.2]. We concentrate on (ii) using the same method going back to Henkin ([18], [19]).

From Lemma 2 it follows that we may assume that $\frac{\partial \varrho}{\partial z_{1}}(z) \neq 0$. Define new coordinates

$$
\begin{aligned}
t_{1}+i t_{2} & =\eta_{1}=\varrho(\zeta)-\varrho(z)+i \operatorname{Im} \Psi(z, \zeta), \\
t_{2 j-1}+i t_{2 j} & =\eta_{j}=\zeta_{j}-z_{j}, \quad j \neq 1,
\end{aligned}
$$

where $\Psi=2 i \psi$ and $\psi$ is the function from Theorem 1 describing the singularities of the Bergman kernel. The Jacobian of this change of coordinates is

$$
\frac{\partial \varrho}{\partial x_{1}} \frac{\partial}{\partial y_{1}} \operatorname{Im} \Psi(z, \zeta)-\frac{\partial \varrho}{\partial y_{1}} \frac{\partial}{\partial x_{1}} \operatorname{Im} \Psi(z, \zeta)
$$

where $\zeta_{j}=x_{j}+i y_{j}, j=1, \ldots, n$. When $z=\zeta$ this expression is equal to

$$
\left(\frac{\partial \varrho}{\partial x_{1}}\right)^{2}+\left(\frac{\partial \varrho}{\partial y_{1}}\right)^{2}
$$

which is not zero by assumption. Consequently, the change of coordinates is valid in some ball $B\left(z, \epsilon^{\prime}\right)$. We can assume that $\epsilon^{\prime}>0$ is independent of $z$. Thus, using Theorem 1 and Lemma 2.1 of [2] we estimate (with $I:=(-1,1)$ )

$$
\begin{aligned}
& \int_{u \cap \Omega} \frac{|K(z, \zeta)|}{|\varrho(\zeta)|^{\alpha}} d V(\zeta)=\left\{\int_{B\left(z, \epsilon^{\prime}\right) \cap \Omega}+\int_{u \cap \Omega \backslash B\left(z, \epsilon^{\prime}\right)}\right\} \frac{|K(z, \zeta)|}{|\varrho(\zeta)|^{\alpha}} d V(\zeta) \\
& \leq C_{\epsilon^{\prime}}+\int_{B\left(z, \epsilon^{\prime}\right) \cap \Omega} \frac{d V(\zeta)}{|\varrho(\zeta)|^{\alpha}\left[\left[|\varrho(z)|+|\varrho(\zeta)|+|\zeta-z|^{2}\right]^{2}+[\operatorname{Im} \Psi(z, \zeta)]^{2}\right]^{(n+1) / 2}} \\
& \leq C_{\epsilon^{\prime}}+C \int_{I^{2 n}} \frac{d t_{1} \cdots d t_{2 n}}{\left|\varrho(z)+t_{1}\right|^{\alpha}\left[\left[\left|\varrho(z)+t_{1}\right|+|\varrho(z)|+t_{1}^{2}+\cdots+t_{2 n}^{2}\right]^{2}+t_{2}^{2}\right]^{(n+1) / 2}} \\
& \leq C_{\epsilon^{\prime}}+C \int_{I} \frac{d t}{|\varrho(z)+t|^{\alpha}\left[|\varrho(z)|+|t+\varrho(z)|+t^{2}\right]} \leq C|\varrho(z)|^{-\alpha} .
\end{aligned}
$$

Proof of Theorem 5. We have to show that for each weight $v$ there exists $w \in W$ and a constant $C$ such that

$$
\|B f\|_{v, \widetilde{u}} \leq C\|f\|_{w, u} .
$$

The inequality for the $L^{2}$ part of the norm is obvious. Consequently, it is enough to construct for a given $v$ a weight $w$ such that

$$
|B f(z)| v(|\varrho(z)|) \leq C\left(\sup _{z \in \mathcal{U} \cap \Omega}|f(z)| w(|\varrho(z)|)+\|f\|_{L^{2}(\Omega)}\right)
$$

for $z \in \widetilde{\mathcal{U}} \cap \Omega$. By Lemma 3(i) we may assume that $v$ is increasing. Let $w$ be defined for $v^{1 / 2}$ as in Lemma 3(ii) with $\alpha=1 / 2$. Let $z \in \widetilde{U}$. Then from 
Lemma 7 it follows that

$$
\begin{aligned}
|B f(z)| \leq & \left\{\int_{\Omega \backslash u}+\int_{u \cap\{|\varrho(\zeta)| \geq|\varrho(z)|\}}+\int_{u \cap\{|\varrho(\zeta)|<|\varrho(z)|\}}\right\}|f(\zeta) K(z, \zeta)| d V(\zeta) \\
\leq & C_{\epsilon} \int_{\Omega}|f(\zeta)| d V(\zeta)+\frac{\|f\|_{w, u}}{v^{1 / 2}(|\varrho(z)|)} \int_{u}|K(z, \zeta)| d V(\zeta) \\
& +\frac{\|f\|_{w, u|\varrho(z)|^{1 / 2}}}{v^{1 / 2}(|\varrho(z)|)} \int_{u} \frac{|K(z, \zeta)|}{|\varrho(\zeta)|^{1 / 2}} d V(\zeta) \\
\leq & C\left(\|f\|_{L^{2}(\Omega)}+\frac{|\log | \varrho(z)||}{v^{1 / 2}(|\varrho(z)|)}\|f\|_{w, u}+\frac{1}{v^{1 / 2}(|\varrho(z)|)}\|f\|_{w, u}\right) .
\end{aligned}
$$

Multiplying both sides by $v(|\varrho(z)|)$ yields the desired conclusion.

Observe that

$$
H W_{\mathfrak{u}}=\bigcup_{n=1}^{\infty} H v_{n, u} .
$$

This can be shown by using the method of proving the algebraic equality of an inductive limit and its projective hull (see [11, direct proof of Theorem 1.3(d)]). Indeed, let $K_{n}$ be compact sets such that $K_{n} \subset \operatorname{int} K_{n+1}$ and $\bigcup_{n=1}^{\infty} K_{n}=(0,1)$. Since the inclusion $H W_{u} \supset \bigcup_{n=1}^{\infty} H v_{n, u}$ is obvious, it is enough to show that for each $f \in H W_{\mathfrak{u}}$ there exists $n \in \mathbb{N}$ such that $\|f\|_{n, u}<\infty$. If this is not the case, then one can find a function $f \in H W_{\mathfrak{u}}$, a strictly increasing sequence of positive integers $a_{n}$ and points $z_{n}$ with $\left|\varrho\left(z_{n}\right)\right| \in \operatorname{int} K_{a(n+1)} \backslash K_{a(n-1)}$ satisfying

$$
v_{n}\left(\left|\varrho\left(z_{n}\right)\right|\right)\left|f\left(z_{n}\right)\right|>n .
$$

Let $\left(\varphi_{k}\right)$ be a continuous partition of unity subordinate to the covering (int $\left.K_{a(n+1)} \backslash K_{a(n-1)}\right)$. Define a weight, as in [11], taking $v_{a}=\sum_{k=1}^{\infty} v_{k-1} \varphi_{k}$. Then $v_{a}$ is a continuous function belonging to the weight family $W$ and

$$
\sup _{z \in \mathcal{U} \cap \Omega}|f(z)| v_{a}(|\varrho(z)|) \geq\left|f\left(z_{n}\right)\right| v_{a}\left(\left|\varrho\left(z_{n}\right)\right|\right) \geq v_{n}\left(\left|\varrho\left(z_{n}\right)\right|\right)\left|f\left(z_{n}\right)\right|>n .
$$

This obviously contradicts the assumption that $f \in H W_{u}$.

From the continuity of $B$ and definitions of the spaces involved it follows that the mapping

$$
L v_{n, u} \rightarrow L W_{u} \rightarrow H W_{\widetilde{u}}
$$

is continuous. Similarly, each inclusion $H v_{n, u} \hookrightarrow H W_{u}$ is continuous.

Consequently, the Grothendieck factorization theorem (see [29]) implies that there exists $m_{n} \in \mathbb{N}$ such that

$$
B\left(L v_{n, u}\right) \subset H v_{m, \widetilde{u}} .
$$

We will show that $m=n+1$. The proof consists of two parts. First, we show that the sequence $m_{n}$ is strictly increasing, and then that $m \leq n+1$. 
The first part amounts to finding a sequence of functions $f_{n} \in L v_{n, u}$ such that $B f_{n} \notin H v_{n, u}$.

It is a classic fact that if $\Omega$ is a $C^{k+2}(k \in \mathbb{N})$ strongly pseudoconvex domain then each point of the boundary is a peak point for the algebra $A(\bar{\Omega})$ ([18], [24]). In other words, there exists a function $\mathcal{P}: \bar{\Omega} \times \partial \Omega \rightarrow \mathbb{C}$ such that for each $\omega \in \partial \Omega$ the function $\mathcal{P}(\cdot, \omega)$ is holomorphic in $\Omega$, continuous in $\bar{\Omega}$ and satisfies

$$
\mathcal{P}(\omega, \omega)=1, \quad|\mathcal{P}(z, \omega)|<1, \quad z \in \bar{\Omega} \backslash\{\omega\} .
$$

Furthermore, $\mathcal{P}(z, \cdot)$ can be constructed to be of class $C^{k}$ for each fixed $z \in \bar{\Omega}$.

We will comment on Henkin's construction of peak functions (see for details [18] and [27]) to draw a somewhat stronger conclusion.

Assume that $\Omega$ is a pseudoconvex domain with $C^{k}$ boundary, $k>2$, and $\varrho$ is a smooth, non-degenerate defining function. Denote by $\mathcal{L}_{\omega}(z)$ the Levi polynomial of $\varrho$ at $\omega$,

$\mathcal{L}_{\omega}(z)=\varrho(\omega)+\sum_{j=1}^{n} \frac{\partial \varrho}{\partial z_{j}}(\omega)\left(z_{j}-\omega_{j}\right)+\frac{1}{2} \sum_{j, k=1}^{n} \frac{\partial^{2} \varrho}{\partial z_{j} \partial z_{k}}(\omega)\left(z_{j}-\omega_{j}\right)\left(z_{k}-\omega_{k}\right)$.

Assume that $\omega \in \mathcal{O}_{\mathrm{s}} \cap \partial \Omega$. Then there exists $\lambda>0$ such that if $|z-\omega|<\lambda$, then

$$
2 \operatorname{Re} \mathcal{L}_{\omega}(z) \leq \varrho(z)-\gamma / 2|z-\omega|^{2},
$$

where $\gamma$ is a non-negative number. Consequently, there exists $\varepsilon>0$ such that $\mathcal{L}_{\omega}(z)<0$ provided $z \in \Omega_{\varepsilon}=\left\{z \in \mathbb{C}^{n}: \varrho(z)<\varepsilon\right\}$ and $|z-\omega| \geq \lambda / 3$. Take a smooth function $\chi: \mathbb{R} \rightarrow[0,1]$ such that $\chi \equiv 0$ for $x \geq 2 \lambda / 3$ and $\chi \equiv 1$ on $[0, \lambda / 3]$.

Observe that the differential form $f_{\omega}$ defined by

$$
f_{\omega}(z)= \begin{cases}-\bar{\partial}_{z}(\chi(|z-\omega|)) \log \mathcal{L}_{\omega}(z), & |z-\omega|<\lambda, z \in \Omega_{\varepsilon} \\ 0, & |z-\omega| \geq \lambda, z \in \Omega_{\varepsilon}\end{cases}
$$

is $C^{\infty}$ and satisfies $\bar{\partial} f_{\omega}=0$ in $\Omega_{\varepsilon}$. Since $\Omega_{\varepsilon}$ is pseudoconvex, there exists a smooth differential form $u_{\omega}$ such that $\bar{\partial} u_{\omega}=f_{\omega}([20])$. The peak function $\mathcal{P}$ is of the form $\mathcal{P}(z, \omega)=\exp (-\Psi(z, \omega))$, where

$$
\Psi(z, \omega)=\exp \left(m u_{\omega}(z)+m \chi(|z-\omega|) \log \mathcal{L}_{\omega}(z)\right) .
$$

Here $m$ is a positive number chosen in such a way that

$$
m\left(\left|\operatorname{Im} u_{\omega}+\chi(|z-\omega|)\right| \operatorname{Im} \log \mathcal{L}_{\omega}(z) \mid\right)<\pi / 2,
$$

which is possible because both $u_{\omega}$ and $\chi(|z-\omega|) \log \mathcal{L}_{\omega}(z)$ have bounded imaginary parts. Indeed, since $\Omega_{\varepsilon}$ is pseudoconvex and $\bar{\Omega}$ is its compact subset, we have

$$
\left\|u_{\omega}\right\|_{L^{\infty}(\Omega)} \leq C\left\|f_{\omega}\right\|_{L_{(0,1)}^{\infty}\left(\Omega_{\varepsilon}\right)}
$$

for some non-negative $C$. 
Importantly, the only point where strong pseudoconvexity is required in the above construction, is the existence of a neighbourhood $U_{\omega}$ of $\omega$ such that (5) holds true. This is a local property of $\partial \Omega$. We can now formulate the conclusion. We use the previously introduced notation.

Proposition 8. Assume that $\Omega$ is a bounded pseudoconvex domain with $C^{3}$ boundary. There exists a function $\mathcal{P}: \Omega \times(\partial \Omega)_{\mathrm{s}} \rightarrow \mathbb{C}$ such that $\mathcal{P}(\cdot, \omega)$ is a peak function for $A(\bar{\Omega})$ for each $\omega \in(\partial \Omega)_{\mathrm{s}}$.

Observe that if $\omega$ belongs to a strongly pseudoconvex piece of $\partial \Omega$, then $\varrho(z) / \mathcal{L}_{\omega}(z)$ is bounded for $z$ in some neighbourhood of $\omega$ in $\Omega$. This can be deduced for example from the Narasimhan lemma (see [27]) or the method of its proof. Indeed, we may assume without loss of generality that $\omega=0$. Consequently, as $\varrho$ can be chosen to be strictly plurisubharmonic in $\omega$ we infer that

$$
\left|\frac{\varrho(z)}{\mathcal{L}_{\omega}(z)}\right|=\left|\frac{2 \operatorname{Re} \mathcal{L}_{\omega}(z)+\sum_{j, k=1}^{n} \frac{\partial^{2} \varrho}{\partial z_{j} \bar{z}_{k}}(\omega) z_{j} \bar{z}_{k}+r_{\omega}(z)}{\mathcal{L}_{\omega}(z)}\right| \leq C
$$

for $z \in \Omega$ sufficiently close to $\omega$. Here $r_{\omega}$ stands for the remainder of order 3 .

Proposition 8 allows us to localize the description of the boundary behaviour of Carathéodory and Kobayashi distances with only slight changes in the original proof (see [1], [24, Theorem (4.5.4)]).

Proposition 9 (Abate [1]). If $\Omega$ is a strongly pseudoconvex domain with $C^{2}$ boundary and $z_{0} \in \Omega$, then

$$
\lim _{z \rightarrow \partial \Omega} \frac{c_{\Omega}\left(z_{0}, z\right)}{-\log \delta_{\Omega}(z)}=\lim _{z \rightarrow \partial \Omega} \frac{d_{\Omega}\left(z_{0}, z\right)}{-\log \delta_{\Omega}(z)}<\infty .
$$

Furthermore, the statement remains valid for a pseudoconvex domain $\Omega$ and $z \rightarrow \partial \Omega \cap \mathcal{U}$.

Proof. Recall ([24, Theorem (4.5.8)]) that on bounded domains in $\mathbb{C}^{n}$ with $C^{2}$ boundary we have

$$
d_{\Omega}\left(z_{0}, z\right) \leq c-\log \delta_{\Omega}(z)
$$

where $c$ depends only on $z_{0}$ and $\Omega$. On the other hand, $c_{\Omega}\left(z_{0}, z\right) \leq d_{\Omega}\left(z_{0}, z\right)$. Thus, to conclude the proof, it is enough to estimate the Carathéodory distance $c\left(z_{0}, z\right)$ from below by $\log \delta_{\Omega}(z)$. Take $z \in U$ and find a point $\omega \in \partial \Omega$ such that $\delta_{\Omega}(z)=|z-\omega|$. Composing the function $\mathcal{P}(\cdot, \omega)$ with the automorphism of $\mathbb{D}$ of the form

$$
\frac{1-\overline{\mathcal{P}\left(z_{0}, \omega\right)}}{1-\mathcal{P}\left(z_{0}, \omega\right)} \cdot \frac{\zeta-\mathcal{P}\left(z_{0}, \omega\right)}{1-\overline{\mathcal{P}\left(z_{0}, \omega\right)} \zeta}
$$

we can assume that $\mathcal{P}\left(z_{0}, \omega\right)=0$. 
Thus from the definition of the Carathéodory distance and properties of the Poincare distance $p$ in the unit disc it follows that

$$
c_{\Omega}\left(z_{0}, z\right) \geq p\left(\mathcal{P}(z, \omega), \mathcal{P}\left(z_{0}, \omega\right)\right) \geq|\log 1-| \mathcal{P}(z, \omega)|| .
$$

Since $u_{\omega}$ is bounded in $\Omega$, we have

$$
\begin{aligned}
\frac{1-|\mathcal{P}(z, \omega)|}{|z-\omega|^{m}} & \leq C \frac{|1-\mathcal{P}(z, \omega)|}{|z-\omega|^{m}} \leq C \frac{|\Psi(z, \omega)|}{|z-\omega|^{m}} \\
& =C\left|\exp u_{\omega}\right| \frac{\left|\exp m \log \mathcal{L}_{\omega}(z)\right|}{|z-\omega|^{m}} \leq C \frac{\left|\mathcal{L}_{\omega}(z)\right|^{m}}{|z-\omega|^{m}} \leq C,
\end{aligned}
$$

provided $|z-\omega| \leq \lambda / 3$. Consequently,

$$
c_{\Omega}\left(z_{0}, z\right) \geq|\log C| z-\left.\omega\right|^{m}|=| \log C \delta_{\Omega}(z)^{m} \mid .
$$

Let $\omega \in \mathcal{O}_{\mathrm{s}}$. As in Lemma 7 we can find a strongly pseudoconvex domain $\Omega_{1}$ such that $\Omega_{1} \subset \Omega$ and $B(\omega, \varepsilon) \cap \partial \Omega=B(\omega, \varepsilon) \cap \partial \Omega_{1}$. Now to obtain a local version of the estimate for the distance $b_{\Omega}$, it suffices to use the theorem of Diederich-Fornæss-Herbort (see [24, Theorem (4.10.25)]). The symbol $d s_{\Omega}^{2}$ stands for the Bergman pseudo-metric.

Theorem 10 (Diederich-Fornæss-Herbort). Let $\Omega$ be a bounded domain of holomorphy in $\mathbb{C}^{n}$, and $z_{0} \in \partial \Omega$. Let $U \subset \subset V$ be small neighbourhoods of $z_{0}$. Then there are positive constants $C, C^{\prime}$ such that

$$
C d s_{\Omega \cap V}^{2} \leq d s_{\Omega}^{2} \leq C^{\prime} d s_{\Omega \cap V}^{2}
$$

on $\Omega \cap U$.

This allows us to conclude (cf. [17, Prop. VII.4.9]) that

$$
\lim _{z \rightarrow \omega \in \mathcal{U} \cap \partial \Omega} \frac{b_{\Omega}\left(z_{0}, z\right)}{\left|\log \delta_{\Omega}(z)\right|}<\infty .
$$

Let $\left(\phi_{i}\right)$ be a smooth partition of unity subordinate to the covering $\left(U_{i}\right)$ from Lemma 2. In [22] we have shown that if $\Omega$ is $C^{1}$ then the Bell operator $([6])$

$$
\Phi h=h-\sum_{j=1}^{n} \partial_{j}\left(\frac{\phi_{j}}{\partial_{j} \varrho} h \varrho\right)
$$

can be extended to an operator $\Phi: H_{1, \varrho}^{2}(\Omega) \cap C_{0, \varrho} \rightarrow L^{2}$ satisfying $B \Phi=$ id on its domain (we refer the reader to $[22]$ for the notation).

Proposition 11. Assume that $\Omega$ is a bounded pseudoconvex domain with $C^{3}$ boundary. Let $\omega \in \partial \Omega$ be a strongly Levi pseudoconvex point and $\mathcal{U}$ an open set containing $\omega$. There exists a sequence of functions $f_{k} \in H v_{k, u} \backslash$ $H v_{k-1, u}$ such that $\Phi f_{k} \in L v_{k-1, u}$.

Proof. Define $f_{k, \omega}(z)=f_{k}(z)=(\log (1-\mathcal{P}(z, \omega)))^{k}$ for $k \in \mathbb{N}$. First of all observe that by the definition of the peak function $\mathcal{P}(\cdot, \cdot), f_{k}$ is a properly 
defined holomorphic function in $\Omega$. For the same reason, we may also restrict our attention to some neighbourhood of $\omega$.

Take $z$ sufficiently close to $\omega$. From the remarks after Proposition 8 and the fact the $u_{\omega}$ is smooth in a neighbourhood of $\Omega$ it follows that

$$
\frac{|\varrho(z)|^{m}}{|\Psi(z, \omega)|^{m}} \leq C \frac{|\varrho(z)|^{m}}{\left|\mathcal{L}_{\omega}(z)\right|^{m}} \leq C .
$$

As a result, $|\log (1-\mathcal{P}(z, \omega))| \leq C|\log | \Psi(z, \omega)|| \leq C|\log | \varrho(z)||$, and consequently $f_{k} \in H v_{k}, u$. The fact that $f_{k}$ does not belong to a space of lower index is obvious. Since near the boundary we have

$$
\Phi h=\sum_{j=1}^{n} \partial_{j}\left(\frac{\phi_{j}}{\partial_{j} \varrho}\right) h \varrho+\frac{\phi_{j}}{\partial_{j} \varrho} \varrho \partial_{j} h,
$$

it is enough to show that $f_{k} \varrho$ is bounded and $\varrho \partial_{j} f_{k}$ belongs to $L v_{k-1, u}$. The first fact follows from the estimate $\left|f_{k}(z)\right||\varrho(z)| \leq C|\log | \varrho(z)||^{k}|\varrho(z)|$. To prove the second observe that

$$
\begin{aligned}
&\left|\partial_{j} f_{k}(z) \varrho(z)\right| \leq C \mid \varrho(z) \frac{f_{k-1}(z)}{1-\mathcal{P}(z, \omega)} \mathcal{P}(z, \omega) \Psi(z, \omega) \mid\left(\left|\partial_{j} u_{\omega}(z)\right|+\left|\frac{C}{\mathcal{L}_{\omega}(z)}\right|\right) \\
& \leq\left|f_{k-1}(z)\right|\left(C+C\left|\frac{\varrho(z) \Psi(z, \omega)}{(1-\mathcal{P}(z, \omega)) \mathcal{L}_{\omega}(z)}\right|\right),
\end{aligned}
$$

since $u_{\omega}$ is smooth in some neighbourhood of $\bar{\Omega}$.

Corollary 12. Assume that $\Omega$ is a bounded strongly pseudoconvex domain in $\mathbb{C}^{n}$ with $C^{3}$ boundary. Then $L W$ is the smallest space containing $L^{\infty}$ defined by weighted-sup seminorms and equipped with the topology given by these seminorms on which $B$ is continuous.

Proof. In view of Theorem 5, it suffices to show that if the Bergman projection is continuous on a space $E$, then the functions defining its seminorms must belong to the weight family $W$.

From the continuity of $B$ we infer that for each $v \in W$ there exists a weight $w$ such that

$$
\left|f_{k}(z)\right| v(|\varrho(z)|)=\left|B \Phi\left(f_{k}\right)(z)\right| v(|\varrho(z)|) \leq C \sup _{z \Omega}\left|\Phi\left(f_{k}\right)(z)\right| w(|\varrho(z)|)
$$

for a positive constant $C$. Consequently, if $\sup _{z \in \Omega}|\log | \varrho(z)||^{k-1} v(|\varrho(z)|)$ $<\infty$ for each $v \in W$, then also $|\log | \varrho(z) \mid{ }^{k} v(|\varrho(z)|)$ must be bounded in $\Omega$ for $v \in W$.

REMARK. Observe that the inequality $|\log | \varrho(z)|| \leq C|\log (1-\mathcal{P}(z, \omega))|$ holds on a set

$$
X(\omega, r)=\left\{z \in \mathbb{C}^{n}:\left|\mathcal{L}_{\omega}(z)\right| \leq r|\varrho(z)|\right\} .
$$


In other words, on this set the function $f_{k, \omega}$ behaves asymptotically exactly like $|\log | \varrho(z)||^{k}$.

Proposition 13. Let $\Omega$ be a bounded, pseudoconvex set with $C^{3}$ boundary. Assume that $E \supset L^{\infty}$ is a space defined by weighted-sup seminorms and equipped with the topology given by these seminorms. If $B$ is continuous on $E$, then $L W \subset E$.

Proof. From the assumptions it follows that the set of strongly pseudoconvex points in $\partial \Omega$ is non-empty. One way to prove this is to recall that by the result in [4] the Shilov boundary of the algebra $A(\bar{\Omega})$ is contained in the closure of the strictly pseudoconvex boundary points of $\Omega$, when $\Omega$ is bounded and has $C^{2}$ boundary. On the other hand, the Shilov boundary of a uniform algebra is always non-empty. Thus, the proof follows from Proposition 11 and the method of the proof of Corollary 12.

Taking into account Corollary 12, it remains to show that $m_{n} \leq n+1$.

Proposition 14. Let $\Omega$ be a bounded, pseudoconvex domain of finite type with smooth boundary. Assume that $z \in \mathcal{U}$, where $\mathcal{U}$ is an open set contained with its closure in $\mathcal{O}_{\mathrm{s}}$. Then

$$
\int_{u \cap \Omega}|\log | \varrho(\zeta)||^{m}|K(z, \zeta)| d V(\zeta) \leq C|\log (|\varrho(z)|)|^{m+1}+C^{\prime}
$$

for positive constants $C, C^{\prime}$.

Proof. Using the method of Lemma 7 we conclude that it is enough to estimate the integral

$$
\begin{aligned}
& \int_{I} \frac{|\log (|t+\varrho(z)|)|^{m}}{|\varrho(z)|+|\varrho(z)+t|+t^{2}} d t \\
& \quad \leq \int_{[-1,|\varrho(z)|]} \frac{|\log (|\varrho(z)|-t)|^{m}}{2|\varrho(z)|-t} d t+\int_{[|\varrho(z)|, 1]} \frac{|\log (t-|\varrho(z)|)|^{m}}{t} d t .
\end{aligned}
$$

Let $\delta>|\varrho(z)|$ and observe that

$$
\begin{aligned}
& \int_{[\delta, 1]} \frac{(-\log (t-|\varrho(z)|))^{m}}{t} d t \\
& \quad=\int_{[\delta, 1]} \frac{(-\log t)^{m}}{t} d t+\int_{[\delta, 1]} \frac{(-\log (t-|\varrho(z)|))^{m}-(-\log t)^{m}}{t} d t \\
& \quad=\frac{1}{m+1}(-\log \delta)^{m+1}+\int_{[\delta, 1]} \frac{(-\log (t-|\varrho(z)|))^{m}-(-\log t)^{m}}{t} d t .
\end{aligned}
$$

It remains to show that the last integral is $O\left(|\log | \varrho(\cdot)||^{m}\right)$. First observe that 


$$
\begin{aligned}
& \left(\log (t-|\varrho(z)|)^{m}=\left[\log t-\sum_{k=1}^{\infty} \frac{|\varrho(z)|^{k}}{k t^{k}}\right]^{m}\right. \\
& =(\log t)^{m}+\sum_{l=0}^{m-1}\left(\begin{array}{c}
m \\
l
\end{array}\right)(-1)^{l}(\log t)^{l} \sum_{n=1}^{\infty}\left(\sum_{j_{1}+\cdots+j_{l}=n} \frac{1}{j_{1} \ldots j_{l}}\right) \frac{|\varrho(z)|^{n}}{t^{n}} .
\end{aligned}
$$

Therefore

$$
\begin{aligned}
& \left|\int_{[\delta, 1]} \frac{(-\log (t-|\varrho(z)|))^{m}-(-\log t)^{m}}{t} d t\right| \\
& \leq|\log \delta|^{m}+\sum_{l=0}^{m-1}\left(\begin{array}{c}
m \\
l
\end{array}\right)|\log | \varrho(z)||^{l} \sum_{n=1}^{\infty}|\varrho(z)|^{n}\left(\sum_{j_{1}+\cdots+j_{l}=n} \frac{1}{j_{1} \cdots j_{l}}\right) \int_{[\delta, 1]} \frac{d t}{t^{n+1}} \\
& \quad \leq|\log \delta|^{m}+\sum_{l=0}^{m-1} 2\left(\begin{array}{c}
m \\
l
\end{array}\right)|\log | \varrho(z)||^{l} \sum_{n=1}^{\infty} \frac{|\varrho(z)|^{n}}{\delta^{n}} \frac{1}{n}\left(\sum_{j_{1}+\cdots+j_{l}=n} \frac{1}{j_{1} \cdots j_{l}}\right)
\end{aligned}
$$

Now, since $|\varrho(z)| / \delta<1$ it is enough to notice that

$$
\sum_{n}^{\infty} \frac{1}{n}\left(\sum_{j_{1}+\cdots+j_{l}=n} \frac{1}{j_{1} \cdots j_{l}}\right) \leq \int_{[1, \infty)^{l}} \frac{d x_{1} \cdots d x_{l}}{\left(\sum_{j=1}^{l} x_{j}\right) x_{1} \cdots x_{l}}<\infty .
$$

The integral

$$
\int_{[-1,|\varrho(z)|]} \frac{(-\log (|\varrho(z)|-t))^{m}}{2|\varrho(z)|-t} d t
$$

can be dealt with in the same manner.

Corollary 15. Let $\Omega$ be a bounded strongly pseudoconvex domain. The Bergman projection is continuous from $L v_{k}(\Omega)$ into $L v_{k+1}(\Omega)$.

Observe that by Proposition 9 and remarks after Theorem 10 it follows that we can define the spaces $L v_{k}$ using the Carathéodory, Kobayashi or Bergman distance from a fixed point rather than $|\log | \varrho(\cdot)||$. This might be a proper language for a possible generalization to a broader class of domains. Now we establish a local version of this result. We keep the notation from the preliminaries.

TheOREM 16. Let $\Omega$ be a bounded pseudoconvex domain of finite type with a smooth boundary. Assume that $\omega_{1}, \ldots, \omega_{k} \in(\partial \Omega)_{\mathrm{s}}$. Then there exist neighbourhoods $\mathcal{U}_{j}$ of $\omega_{j}, j=1, \ldots, k$, such that for each $f \in L^{2}(\Omega)$ satisfying

$$
|f(z)| \leq C b_{\Omega}\left(z, z_{0}\right)^{m_{j}}, \quad z \in \mathcal{U}_{j} \cap \Omega,
$$

where $m_{j} \in \mathbb{N}_{0}$, we have

$$
|B f(z)| \leq C^{\prime} b_{\Omega}\left(z, z_{0}\right)^{m_{j}+1}, \quad z \in \widetilde{\mathcal{U}}_{j} \cap \Omega,
$$

where $\widetilde{\mathcal{U}}_{j} \subset \subset \mathcal{U}_{j}$. 
Proof. It is enough to notice that if $\omega$ is a strongly pseudoconvex point in $\partial \Omega$, then there exists a defining function $\varrho$ which is strictly plurisubharmonic in some neighbourhood of $\omega$ (cf. the proof of the Narasimhan lemma in [27]). The assertion now follows from Proposition 14 and Theorem 10.

To complete the picture of the boundary behaviour of the Bergman projection define

$$
H_{v_{n}}^{1}(\Omega)=\left\{f \in H(\Omega): \int_{\Omega}|f(\zeta)| v_{n}^{-1}(|\varrho(\zeta)|) d V(\zeta)<\infty\right\},
$$

and let $H_{v}^{1}$ denote the projective limit of the sequence

$$
\cdots \rightarrow H_{v_{n}}^{1} \rightarrow \cdots \rightarrow H^{1} .
$$

The symbols $L_{v_{n}}^{1}$ and $L_{v}^{1}$ will denote the corresponding spaces of measurable functions.

The proof of the next proposition is exactly the same as in the case of the unit ball (see [22]).

Proposition 17. The space $H W$ is isomorphic to the strong dual of $H_{v}^{1}$. The space $H_{v}^{1}$ is a Fréchet-Schwartz space.

By duality, we obtain

Proposition 18. The Bergman projection is continuous on $L_{v}^{1}$.

OPEn PROBLEM. The results of this paper suggest the question whether Theorem 16 as well as its global analog hold true for any bounded pseudoconvex domain in $\mathbb{C}^{n}$, or at least a pseudoconvex domain of finite type near any boundary point. This is closely connected with understanding the singularities of the Bergman kernel. It is not clear whether one can expect to obtain estimates with $\left|\log \operatorname{dist}_{\Omega}(z)\right|$. As already pointed out, this is closely connected with the problem of finding a suitable extension of $L^{\infty}$ on which $B$ is bounded.

Acknowledgements. The author wishes to thank Prof. E. Ligocka for crucial suggestions.

\section{References}

[1] M. Abate, Boundary behaviour of invariant distances and complex geodesics, Rend. Accad. Naz. Lincei 80 (1986), 100-106.

[2] P. R. Ahern and R. Schneider, Holomorphic Lipschitz functions in pseudoconvex domains, Amer. J. Math. 101 (1979), 543-565.

[3] D. E. Barrett, Regularity of the Bergman projection and local geometry of domains, Duke Math. J. 53 (1986), 333-343.

[4] R. F. Basener, Peak points, barriers and pseudoconvex boundary points, Proc. Amer. Math. Soc. 65 (1977), 89-92. 
[5] S. R. Bell, The Cauchy Transform, Potential Theory, and Conformal Mapping, CRC Press, Boca Raton, 1992.

[6] -, Biholomorphic mappings and the $\bar{\partial}$-problem, Ann. of Math. 114 (1981), 103-113.

[7] S. R. Bell and H. P. Boas, Regularity of the Bergman projection, Math. Ann. 267 (1984), 473-478.

[8] - - - Regularity of the Bergman projection in weakly pseudoconvex domains, ibid. 257 (1981), 23-30.

[9] S. R. Bell and E. Ligocka, A simplification and extension of Fefferman's theorem on biholomorphic mappings, Invent. Math. 57 (1980), 283-289.

[10] K. D. Bierstedt, An introduction to locally convex inductive limits, in: Functional Analysis and its Applications (Nice, 1996), World Sci., 1988, 35-133.

[11] K. D. Bierstedt, R. Meise and W. H. Summers, A projective description of weighted inductive limits, Trans. Amer. Math. Soc. 272 (1982), 107-160.

[12] L. Boutet de Monvel et J. Sjöstrand, Sur la singularité des noyaux de Bergman et Szegö, Astérisque 34-35 (1976), 123-164.

[13] M. Engliš, A Forelli-Rudin construction and asymptotics of weighted Bergman kernels, J. Funct. Anal. 177 (2000), 257-281.

[14] M. Engliš, T. Hänninen and J. Taskinen, Minimal $L^{\infty}$-type spaces on strictly pseudoconvex domains on which the Bergman projection is continuous, preprint.

[15] C. Fefferman, The Bergman kernel and biholomorphic mappings of pseudoconvex domains, Invent. Math. 16 (1974), 1-65.

[16] F. Forelli and W. Rudin, Projections on spaces of holomorphic functions in balls, Indiana Univ. Math. J. 24 (1974), 593-602.

[17] K. Fritzsche and H. Grauert, From Holomorphic Functions to Complex Manifolds, New York, 2002.

[18] G. M. Henkin, Integral representations of functions holomorphic in strictly pseudoconvex domains and some applications, Math. USSR-Sb. 7 (1969), 597-616.

[19] G. M. Henkin and J. Leiterer, Theory of Functions on Complex Manifolds, Akademie-Verlag, Berlin, 1984.

[20] L. Hörmander, $L^{2}$ estimates and existence theorems for the $\bar{\partial}$ operator, Acta Math. 113 (1965), 89-152.

[21] M. Jarnicki and P. Pflug, Invariant Distances and Metrics in Complex Analysis, de Gruyter, Berlin, 1993.

[22] M. Jasiczak, On locally convex extension of $H^{\infty}$ in the unit ball and continuity of the Bergman projection, Studia Math. 156 (2003), 261-275.

[23] N. Kerzman, The Bergman kernel function. Differentiability at the boundary, Math. Ann. 195 (1972), 149-158.

[24] S. Kobayashi, Hyperbolic Complex Spaces, Springer, Berlin, 1998.

[25] J. J. Kohn, Boundary behavior of $\bar{\partial}$ on weakly pseudoconvex manifolds of dimension two, J. Differential Geom. 6 (1972), 523-542.

[26] -, Subellipticity of the $\bar{\partial}-$ Neumann problem on pseudoconvex domains: sufficient conditions, Acta Math. 142 (1979), 79-122.

[27] S. G. Krantz, Function Theory of Several Complex Variables, Wiley, New York, 1982.

[28] -, Geometric Analysis and Function Spaces, CBMS Reg. Conf. Ser. Math. 81, Amer. Math. Soc., Providence, RI, 1991.

[29] R. Meise and D. Vogt, Introduction to Functional Analysis, Oxford Univ. Press, Oxford, 1997. 
[30] J. Taskinen, On the continuity of the Bergman and Szegö projections, Houston J. Math. 30 (2004), 171-190.

Faculty of Mathematics and Computer Science

Adam Mickiewicz University

Umultowska 87

61-614 Poznań, Poland

E-mail:mjk@amu.edu.pl

Received July 17, 2003

Revised version June 12, 2004 